\title{
Prophylactic Effect of Tranexamic Acid on Hemorrhage During and After the Cesarean Section
}

\author{
Forozan Milani $^{\circledR}$, Katayoun Haryalchi ${ }^{2 *}{ }^{\mathbb{D}}$, Seyedeh Hajar Sharami ${ }^{1}$, Zahra Atrkarroshan ${ }^{3}$, Sara Farzadi $^{1}$
}

\begin{abstract}
Objectives: Recent guidelines of the World Health Organization (WHO) indicated administering tranexamic acid (TXA) in order to treat postpartum hemorrhage (PPH). Therefore, finding low-cost and low-risk alternative methods to control obstetric hemorrhage is of great importance. The present study aimed to evaluate the prophylactic effect of TXA on hemorrhage during and after the cesarean section (CS). In addition, it was attempted to explore the impact of TXA as a safe and inexpensive method for decreasing bleeding during and after CS so that to decrease the hazard of blood transfusion or hysterectomy in these patients.

Materials and Methods: This randomized double-blind control trial was performed on 60 women who underwent CS using spinal anesthesia. These women were randomly assigned to two groups. The experimental group received $1 \mathrm{~g}$ of TXA 15 minutes before the incision while the control group received dextrose $5 \%$ in water as a placebo. The amount of bleeding was measured during and after the surgery. Data were entered into SPSS software version 21 and reported by descriptive statistics and analyzed using chi-square, $t$ test, Mann-Whitney $\mathrm{U}$ test, paired $t$ test, and ANCOVA and Wilcoxon tests.

Results: Based on the results, no significant difference was found between the groups regarding the mean age, mean gestational age, and the prothrombin time (PT) and partial thromboplastin time (PTT) $(P>0.05)$. The mean of the total bleeding volume was 551.8 and $713.1 \mathrm{~mL}$ for experimental and control groups, respectively which means that a significant difference was observed between both groups in this respect $(P=0.006)$. However, no significant difference was noted between the groups regarding changes in hemoglobin $(\mathrm{Hb})$ concentration, systolic and diastolic blood pressure (BP), and heart rate.

Conclusions: Generally, administering $1 \mathrm{mg}$ of TXA in CS significantly reduced the volume of PPH. However, it did not cause significant changes in hemodynamic state or $\mathrm{Hb}$ level. Therefore, it can be recommended as an appropriate treatment for these patients.
\end{abstract}

Keywords: Tranexamic acid, Bleeding, Caesarian secession

\section{Introduction}

Nearly 530000 maternal deaths annually occur due to pregnancy and childbirth worldwide. Obstetric bleeding which often happens after the delivery is one of the chief reason for maternal mortality (1). Based on recent reports, cesarean delivery causes more bleeding than vaginal delivery (2). The World Health Organization (WHO) defined postpartum hemorrhage $(\mathrm{PPH})$ as $>500 \mathrm{~mL}$ blood loss from the canal of birth in 24 hours after birth (3). It is inevitable to prevent $\mathrm{PPH}$, particularly after cesarean delivery. Nowadays, systemic antifibrinolytic agents are increasingly administered during the surgery in order to decrease the volume of blood loss and prevent fibrinolysis, namely, dissolution of the blood clot. Numerous studies were conducted regarding the prophylactic impact of administering tranexamic acid (TXA) on bleeding throughout the surgery and consequently less need for blood transfusions during anesthesia in liver transplantation and spinal, urologic, orthopedics, and cardiac surgeries (4).

Fibrinogen and fibrin quickly dissolve as the placenta is expulsed during the cesarean delivery. After placental expulsion, activation of fibrinolytic system prompted clinicians to apply TXA. Meanwhile, activating the fibrinolytic system can increase the activators of plasminogen and fibrin degradation products (FDP). In addition, fibrinolytic activation system may endure 6 to 12 hours, causing further bleeding.

Since TXA is an antifibrinolytic drug (4), recent guidelines of the WHO recommended administering TXA for PPH treatment if oxytocin and uterotonics were ineffective to halt the bleeding or trauma as the potential causes of bleeding. As the WHO noted the need for a modified guideline consequent to further researches (5), more in-depth studies are suggested about the effect of TXA on controlling the PPH. Further, the WHO and the Iranian ministry of health and medical education (MoHME) have made huge investments in preventing maternal mortality. Hence, it is crucial to identify low-cost and low-risk alternative methods of controlling obstetric hemorrhage. Therefore, the current study sought to investigate the prophylactic effect of TXA on hemorrhage 
during and after the cesarean section (CS). Furthermore, the impact of TXA as a safe and inexpensive method to reduce bleeding during and after CS was assessed to decrease the risk of blood transfusion or hysterectomy in these patients.

\section{Materials and Methods}

This is a randomized, double-blind clinical trial which was conducted on 60 primipara or multipara singleton pregnant women at 37-40 weeks of gestation and ASA (American Society of Anesthesiologists) class I and II. They were admitted to Al-Zahra hospital of Rasht (located in north part of Iran) during 2015-2016. The pregnant women had cesarean delivery under spinal anesthesia (6). The exclusion criteria encompassed patients:

- Suffering from medical diseases including liver, heart, kidney, brain, and blood disorders;

- Having a history of thromboembolic disease, abnormal placenta, severe preeclampsia, multiple pregnancies (multipara), macrosomia, and polyhydramnios;

- Required blood transfusion due to anemia;

- Having an adverse gynecologic history (i.e., abortion, intra-uterine growth retardation, and the like);

- Suffering from rheumatic diseases, obesity;

- Having symptoms of deep vein thrombosis, varicose veins, or rupture of membrane.

Additionally, the exclusion criteria were applied to patients with abnormal results from coagulation tests including prothrombin time (PT) and partial thromboplastin time (PTT) before administering the drug. In addition, allergy to the TXA and patient dispensing were considered the other reasons for exclusion. The patients were selected through block randomization. In the operating room, A and B packages were offered to the patients in order to make a choice, which only the nurse responsible for preparing the drug was aware of the content. All drugs were administered by an anesthesiologist who was unaware of managing and evaluating the patients. Both groups underwent spinal anesthesia with lidocaine 5\% (Shahid-Ghazi, Tabriz, Iran) and were injected with $20 \mu \mathrm{g}$ of fentanyl (Abureihan, Tehran, Iran). The TXA (Kaspian, Rasht, Iran) was colorless and looked similar to distilled water (DW) Therefore, the anesthesiologist was not aware of the type of the administered drug (either TXA or DW).

The experimental group received 1 gram of intravenous TXA [diluted in $20 \mathrm{cc}$ of DW 5\%] exactly 15 minutes before the skin incision while the control group was injected with $10 \mathrm{cc}$ of DW [Diluted in $20 \mathrm{cc}$ of DW 5\%] as a placebo. After the delivery, each group received 1 liter of ringer lactate with 30 units of oxytocin (Kaspian, Rasht, Iran). Measuring blood loss was started after placental expulsion up to the completion of surgery (T1) and from the completion of CS up to 2 hours after the delivery (T2). The cesarean pack (it refers to a set of sterilized tools and drapes used for cesarean delivery) was weighed by a scale before the operation. All gauzes which were applied to dry the surgical zone were precisely counted at the beginning of the sections. All sections were performed with Pfannenstiel incision. The sterile drapes overlying the patient, which were included in the cesarean pack, were stitched up again and then weighed with the same scale. To isolate the amniotic fluid from intraoperative bleeding, when the uterus was cut and the amniotic sac was ruptured, it was collected in another container. Then, the placenta was connected to another adjacent container to collect the blood immediately after the expulsion. The volume of blood in the second container was considered as the intraoperative blood loss. At the end of the operation and when the patient was transferred to the recovery room, all pads were collected and weighed for 2 hours. Based on the total blood of the suction container during and after the cesarean delivery, the volume of blood loss was calculated after the expulsion of the placenta. Further, the gauzes and sterile drapes covering the patient's body during the surgery were computed using the following equation. Then, both groups were compared regarding the volume of blood loss during and 2 hours after the operation

The patient's vital signs including blood pressure (BP), pulse rate $(\mathrm{PR})$, and base hemoglobin $(\mathrm{Hb})$ were measured before the $\mathrm{CS}$. The $\mathrm{Hb}$ was routinely measured again within 12 to 24 hours after the operation in the hospital. In this study, $\mathrm{Hb}, \mathrm{PR}$, and $\mathrm{BP}$ of the patients were recorded within 12 to 24 hours after the operation.

\section{Statistical Analysis}

The SPSS software, version 21.0 (SPSS Inc., Chicago, IL, USA) was used for statistical analysis. After collecting and recording the data, $t$ test and paired $t$ test were adopted based on the research objectives in case the data distribution was normal. Otherwise, a similar nonparametric test was employed.

\section{Results}

Totally, mean age of the women receiving TXA and placebo was $29.33 \pm 5.59$ and $31.2 \pm 5.53$, respectively. Based on the results, no significant difference was observed between the groups regarding the age $(P=$ 0.199 ). Furthermore, there was no significant difference between the women aged $<$ and $>30$ years in term of mean age. Moreover, no significant differences were found between the experimental and control groups respecting the mean age groups, mean gestational age, mean PT, and PTT (Table 1). The results indicated that the volume of blood collected during the cesarean delivery for the experimental group was $140 \mathrm{~mL}$ less than that of the placebo-receiving group. This difference was statistically significant. Additionally, the volume of blood collected 2 hours after the cesarean delivery indicated a difference as limited as $21 \mathrm{cc}$, which was also statistically significant. In 
Table 1. Mean Gestational Age and Coagulation Factors in the 2 Groups

\begin{tabular}{lccc}
\hline & Control & Experimental & P Value \\
\hline Mean gestational age (wk) & $37.86 \pm 0.81$ & $37.93 \pm 0.69$ & 0.735 \\
Mean prothrombin time (s) & $12.04 \pm 0.75$ & $11.95 \pm 0.51$ & 0.73 \\
Mean partial & $31.73 \pm 3.1$ & $32.03 \pm 4.01$ & 0.747 \\
thromboplastin time (s) & & & \\
\hline
\end{tabular}

addition, significant differences were observed in the total volumes of blood during and two hours after the surgery. Further, higher blood loss was noted in the placebo group (Table 2).

The results demonstrated no significant difference between the groups regarding pre and postoperative $\mathrm{Hb}$ levels $(P=0.236 \& P=0.818)$. The preoperative levels were $12.41 \pm 1.16$ and $12.77 \pm 1.13$ in the intervention and control groups, respectively whereas the postoperative levels were $11.78 \pm 1.0$ and $11.7 \pm 1.69$, respectively. Furthermore, there was no statistically significant difference between pre and postoperative Hb levels $(P=0.11)$.

As regards systolic BP, no significant difference was observed between the groups during and after the cesarean delivery. Additionally, although there was a significant difference between diastolic BP during and after the cesarean delivery in each group, no such difference was found between the two groups in each time period. In addition, considering the HR changes after the surgeries as well as HR changes in individual periods before and after the operations there were no statistically significant changes between the groups. Similarly, no significant difference was observed between the groups with regard to the mean reduction of blood $\mathrm{Hb}$ before and after the cesarean delivery (Table 3).

\section{Discussion}

The PPH which is an early complication of cesarean delivery is defined as $>500 \mathrm{~mL}$ and $>1000 \mathrm{~mL}$ of blood losses after vaginal and cesarean delivery, respectively with a hematocrit drop $>10 \%(7-9)$. It is the main reason for maternal mortality in low-income countries. Further, it is the primary cause of nearly $1 / 4$ of maternal mortalities worldwide. The results revealed that using timely prophylactic uterotonics could be helpful (5) and that TXA as an antifibrinolytic drug could halt the bleeding.

Furthermore, the results indicated that administering TXA during cesarean delivery could curtail postpartum bleeding. The mean and the total blood volume collected during and 2 hours after the operations were significantly different between the groups $(P=0.017, P=0.038$, and $P$ $=0.006$, respectively).

A number of studies were conducted in this respect. $\mathrm{Xu}$ et al, for example, found no significant difference regarding the volumes of blood loss from placental expulsion up to the end of CS. However, a lower amount of blood loss was observed in the TXA group after cesarean until 2 following hours. Regarding the total blood loss from placental expulsion up to 2 hours after the cesarean delivery, a significant difference was detected between the TXA and placebo groups. This result is in line with the fining of the current study which demonstrated a lower blood loss in the TXA group (3). It should be noted that Xu et al administered the medication based on the patient's weight while in this study $1 \mathrm{~g}$ of TXA was administered to all the patients. Similarly, Gungorduk et al administered $1 \mathrm{~g}$ of TXA to the patients and obtained similar results indicating that administering TXA to the experimental group could curtail blood loss by $17 \%$ compared to the control group (10). Moreover, in a multicenter study, Gai et al found that the TXA-receiving group had a lower blood loss (18\%) compared to the control group (8). Additionally, in a meta-analysis performed by Peitsidis \& Kadir and Novikova et al concluded that TXA could significantly reduce the blood loss compared to the placebo (11-13). Nevertheless, Novikova et al conducted a new study and indicated that TXA could be helpful in patients with a low risk of bleeding (13). Similar to Xu et al, Movafegh et al obtained similar results by using the

Table 2. Mean Blood Collected During and 2 Hours After Operation in the 2 Groups

\begin{tabular}{|c|c|c|c|c|}
\hline & Control & Experimental & $T$ Value & $P$ Value \\
\hline Mean blood collected during operation $(\mathrm{mL})$ & $649.9 \pm 234.1$ & $509.5 \pm 208.7$ & 2.45 & 0.017 \\
\hline Mean blood collected $2 \mathrm{~h}$ after operation $(\mathrm{mL})$ & $63.2 \pm 48.84$ & $42.3 \pm 21.75$ & 2.14 & 0.038 \\
\hline Mean blood collected during and $2 \mathrm{~h}$ after operation $(\mathrm{mL})$ & $713.1 \pm 233.1$ & $551.8 \pm 207.8$ & 2.82 & 0.006 \\
\hline
\end{tabular}

Table 3. Mean Vital Signs of Patients Before and After Operation

\begin{tabular}{|c|c|c|c|c|c|}
\hline & & Control & Experimental & Z Value & $P$ Value \\
\hline \multirow{2}{*}{ Systolic blood pressure variations } & Preoperative & $114.66 \pm 9.73$ & $114.33 \pm 6.26$ & 1.91 & 0.849 \\
\hline & Postoperative & $111.66 \pm 6.47$ & $111.83 \pm 6.36$ & 0.218 & 0.827 \\
\hline \multirow{2}{*}{ Diastolic blood pressure variations } & Preoperative & $73.5 \pm 7.78$ & $73.5 \pm 5.43$ & 0.15 & 0.877 \\
\hline & Postoperative & $69.66 \pm 5.56$ & $69.66 \pm 6.14$ & 0.159 & 0.874 \\
\hline \multirow{2}{*}{ Heart rate Per minute variations } & Preoperative & $82.1 \pm 6.06$ & $83.46 \pm 3.31$ & 1.44 & 0.148 \\
\hline & Postoperative & $85.56 \pm 5.83$ & $83.06 \pm 5.21$ & 1.48 & 0.138 \\
\hline
\end{tabular}


drug based on the patients' weight. Based on the results, a lower mean amount of blood loss was found in the TXA group compared to control one in terms of intraoperative $(404.7 \pm 94.4 \mathrm{~mL}$ vs. $262.5 \pm 39.6 \mathrm{~mL})$ and postoperative blood loss $(P<0.001,141.0 \pm 33.9 \mathrm{~mL}$ vs. $67.1 \pm 6.5 \mathrm{~mL})$ (14). In addition, a recent systematic review demonstrated that comparing mean reduction in blood loss volume were respectively $141.25 \mathrm{~mL}$ (95\% CI: -186.72 to -95.79 ; $I^{2}=99 \%$ ) and $22.88 \mathrm{~mL}$ (95\% CI: -50.54 to $\left.4.77 ; I^{2}=0 \%\right)$ administering TA in CS and vaginal delivery to control group (14).

Likewise, Li et al found significantly less blood loss during the intraoperative and postoperative periods in patients receiving TXA compared to that of the placebo group (15).

Similar to this study, $\mathrm{Xu}$ et al reported there was no significant difference between the two groups in terms of $\mathrm{Hb}$ levels. However, based on the results, $\mathrm{Hb}$ reduction was more intense in the TXA group compared to that of the control group (3). Further, Movafegh et al presented similar results indicating that postoperative systolic and diastolic BP were identical in both groups $(P<0.05)(18)$.

\section{Conclusions}

Generally speaking, it was revealed that $1 \mathrm{~g}$ of TXA administered to the pregnant women undergoing cesarean delivery could significantly reduce the volume of PPH. However, it did not cause variations in hemodynamic and $\mathrm{Hb}$ levels. It was concluded that TXA, which is strongly recommended by the $\mathrm{WHO}$, plays a beneficial role in preventing $\mathrm{PPH}$ and maternal mortality. As postpartum bleeding is a disastrous reason for maternal mortality, it is essential to perform further investigations including more inclusive statistical populations and a larger sample size.

\section{Conflict of Interests}

Authors declare that they have no conflict of interests.

\section{Ethical Issues}

The trial was approved by the Ethical Committee of Guilan University of Medical Sciences (ethics No. 1930119605 dated: 2014.06.14) and all the women signed the written informed consent. Moreover, the IRCT code assigned was IRCT201405313485N4.

\section{Financial Support}

This study was financially supported by the Vicechancellorships of Research Center in Guilan University of Medical Sciences.

\section{Acknowledgments}

The authors appreciate the financial support provided by the Vice-chancellor of the Research Center in Guilan University of Medical Sciences. Furthermore, this investigation was based on a thesis submitted by the fifth author to the Guilan University of Medical Sciences in
Rasht, Iran.

\section{References}

1. Shakur H, Elbourne D, Gulmezoglu M, et al. The WOMAN Trial (World Maternal Antifibrinolytic Trial): tranexamic acid for the treatment of postpartum haemorrhage: an international randomised, double blind placebo controlled trial. Trials. 2010;11:40. doi:10.1186/1745-6215-11-40

2. Mayur G, Purvi P, Ashoo G, Pankaj D. Efficacy of tranexamic acid in decreasing blood loss during and after cesarean section: a randomized case controlled prospective study. J Obstet Gynaecol India. 2007;57(3):227-30.

3. Xu J, Gao W, Ju Y. Tranexamic acid for the prevention of postpartum hemorrhage after cesarean section: a double-blind randomization trial. Arch Gynecol Obstet. 2013;287(3):463-468. doi:10.1007/s00404-012-2593-y

4. Weeks A. The prevention and treatment of postpartum haemorrhage: what do we know, and where do we go to next? BJOG. 2015;122(2):202-210. doi:10.1111/14710528.13098

5. World Health Organization )WHO). WHO recommendations for the prevention and treatment of postpartum haemorrhage. WHO; 2012.

6. Miller RD, Eriksson LI, Fleisher LA, Wiener-Kronish JP, Cohen NH, Young WL. Miller's Anesthesia. Elsevier Health Sciences; 2014.

7. Nguyen-Ky T, Wen PP, Li Y. Consciousness and depth of anesthesia assessment based on Bayesian analysis of EEG signals. IEEE Trans Biomed Eng. 2013;60(6):1488-1498. doi:10.1109/tbme.2012.2236649

8. Gai MY, Wu LF, Su QF, Tatsumoto K. Clinical observation of blood loss reduced by tranexamic acid during and after caesarian section: a multi-center, randomized trial. Eur J Obstet Gynecol Reprod Biol. 2004;112(2):154-157. doi:10.1016/S0301-2115(03)00287-2

9. Yang $\mathrm{H}$, Zheng S, Shi C. [Clinical study on the efficacy of tranexamic acid in reducing postpartum blood lose: a randomized, comparative, multicenter trial]. Zhonghua $\mathrm{Fu}$ Chan Ke Za Zhi. 2001;36(10):590-592.

10. Gungorduk K, Yildirim G, Asicioglu O, Gungorduk OC, Sudolmus S, Ark C. Efficacy of intravenous tranexamic acid in reducing blood loss after elective cesarean section: a prospective, randomized, double-blind, placebocontrolled study. Am J Perinatol. 2011;28(3):233-240. doi:10.1055/s-0030-1268238

11. Peitsidis P, Kadir RA. Antifibrinolytic therapy with tranexamic acid in pregnancy and postpartum. Expert Opin Pharmacother. 2011;12(4):503-516. doi:10.1517/146 56566.2011.545818

12. Novikova N, Hofmeyr GJ. Tranexamic acid for preventing postpartum haemorrhage. Cochrane Database Syst Rev. 2010(7):Cd007872. doi:10.1002/14651858.CD007872.pub2

13. Novikova N, Hofmeyr GJ, Cluver C. Tranexamic acid for preventing postpartum haemorrhage. Cochrane Database Syst Rev. 2015(6):Cd007872. doi:10.1002/14651858. CD007872.pub3

14. Chattopadhyay S, Sarkar S, Chakrabarti S, Mandal MC. Effect of Intravenous Tranexamic Acid Administration on Blood Loss During and After Elective Caesarean Delivery-A Randomised Placebo-Controlled Study. 
Journal of Evolution of Medical and Dental Sciences. 2017;6(16):1286-1292. doi:10.14260/Jemds/2017/279

15. Li C, Gong Y, Dong L, Xie B, Dai Z. Is prophylactic tranexamic acid administration effective and safe for postpartum hemorrhage prevention?: A systematic review and meta-analysis. Medicine (Baltimore). 2017;96(1):e5653. doi:10.1097/md.0000000000005653

16. O’Brien KL. Evidence for/Against Administration of Antifibrinolytic Agents During an Obstetrical Hemorrhage.
Springer; 2018:47-54. doi:10.1007/978-3-319-77140-3_5

17. Alavi A, Atiqpour F, Zare S, Nasiri Z. Effect of tranexamic acid on postpartum vaginal hemorrhage in Sharieti Hospital of Bandar Abbas in 2014-2015. J Res Med Dent Sci. 2018;6(3):452-458. doi:10.24896/jrmds.20186369

18. Movafegh A, Eslamian L, Dorabadi A. Effect of intravenous tranexamic acid administration on blood loss during and after cesarean delivery. Int J Gynaecol Obstet. 2011;115(3):224-226. doi:10.1016/j.ijgo.2011.07.015

(c) 2019 The Author (s); This is an open-access article distributed under the terms of the Creative Commons Attribution License (http://creativecommons.org/licenses/by/4.0), which permits unrestricted use, distribution, and reproduction in any medium, provided the original work is properly cited. 